\title{
Differential 8-APSK monolithically integrated Dual- EML transmitter for flexible coherent PONs
}

\author{
J. Camilo Velásquez, ${ }^{1,}{ }^{*}$ Jeison Tabares, ${ }^{1}$ And Josep Prat ${ }^{1}$ \\ ${ }^{1}$ Signal Theory and Communications Department, Universitat Politècnica de Catalunya (UPC), Jordi Girona 31., Barcelona, Spain. 08034 \\ *Corresponding author: juavelasquez@tsc.upc.edu
}

Received XX Month XXXX; revised XX Month, XXXX; accepted XX Month XXXX; posted XX Month XXXX (Doc. ID XXXXX); published XX Month XXXX

\begin{abstract}
We experimentally demonstrate a simultaneous amplitude and phase modulation of a monolithically integrated dual electro-absorption modulated laser (DEML). The proposed technique combines a 4-ary direct phase modulation with a 2-level amplitude modulation obtaining an 8-ary amplitude-phase shift keying (8APSK) external-modulator-free transmitter. Its performance was tested up to $7.5 \mathrm{~Gb} / \mathrm{s}$ in a $25-\mathrm{km}$ single mode fiber link with intradyne coherent detection. A

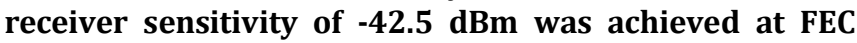
limit BER $=4 \times 10^{-3}$. The results show that the proposed system can be an efficient flexible transmitter for next generation passive optical networks.
\end{abstract}

http://dx.doi.org/10.1364/OL.99.099999

A large list of versatile services with often diverging requirements is expected to arise with the $5 \mathrm{G}$ era, facing new challenges to the telecommunication industry, where low complexity and cost are preferred [1]. Coherent transceivers (TRx) with high aggregate capacity and enhanced spectral efficiency have been demonstrated using bulk devices [2]. However their complexity and cost have limited their feasibility as a potential solution, especially in access networks. To face this issue, low-cost transceivers were presented in a field-trial demonstration [3]. In addition, the hardware devices should support flexible transmission capacity and simultaneously have a low energy consumption [4]. Therefore, an important factor to be considered is the TRx footprint.

Recently we presented a novel application of a monolithically integrated dual electro-absorption modulated laser (DEML), showing a simultaneous differential phase shift keying (DPSK) and amplitude shift keying (ASK) modulation. An optical signal format DPSK/ASK was generated, transmitted and detected with a heterodyne coherent receiver ( $\mathrm{Rx})$ [5].

In this work we extend our research, demonstrating for the first time to our knowledge, a simultaneous differential quadrature shift keying (DQPSK)/ASK or differential 8-APSK modulation, through the same DEML. The primary objectives are to maximize the spectral efficiency, increase the bitrate $(\mathrm{Rb})$ and improve the $\mathrm{Rx}$ sensitivity with respect to [5] using an intradyne coherent $\mathrm{Rx}$.
The complete transmitter (Tx) was based on a DEML whose two sections (DFB and EAM) were independently modulated with different data streams. For the DFB, the quaternary data to generate the DQPSK signal were composed by two uncorrelated PRBS sequences $\left(d_{1}(t)\right.$ and $\left.d_{2}(t)\right)$. The symbols were codified as a 4 -ary signal with four different phase variations $(0, \pm \pi / 2, \pi)$, mapped into a multi-level signal and differentially encoded for direct phase modulation of the laser [6]. The symbol sequence was digitally equalized by means of a 1-tap finite impulse response (FIR) filter with high-pass response. The equalized electrical waveform generates a phase modulated signal by differentiating the frequency variations of the directly modulated laser produced by the adiabatic chirp. This frequency deviation is proportional to the modulating current. Therefore, we can control the phase changes of the optical signal adjusting the duty cycle and amplitude of the dicode return to zero (RZ) shaped modulating waveform [7]. For the EAM, the data to produce the ASK signal were a third PRBS sequence $\left(d_{3}(t)\right)$ coded as non-return to zero (NRZ) and uncorrelated with the data used to modulate the DFB. Both symbol sequences were uploaded to a $5 \mathrm{GSa}$ /s arbitrary waveform generator (AWG) and digitally synchronized. The AWG produced two electrical signals at 2.5 Gbaud, one for the DFB $(R b=$ $5 \mathrm{~Gb} / \mathrm{s})$ and other for the EAM $(R b=2.5 \mathrm{~Gb} / \mathrm{s})$. The amplitude of both electrical signals was tuned with two identical $10 \mathrm{GHz}$ BW electrical amplifiers and injected to the corresponding sections of the DEML. The result was an optical 8-APSK signal with four orthogonal phases $\left(\varphi_{0}(t), \varphi_{1}(t), \varphi_{2}(t), \varphi_{3}(t)\right)$ and two possible amplitudes $\left(a_{0}(t), a_{1}(t)\right)$ at $R b=7.5 \mathrm{~Gb} / \mathrm{s}$. The setup and constellation diagram of the 8-APSK signal are shown in Fig. 1.

The DEML, used at the $\mathrm{Tx}$, is a monolithically integrated photonic device where the same active layer is composed by two sections, a distributed feedback (DFB) laser and an electroabsorption modulator (EAM) [8], in our case, with lengths of 470 $\mu \mathrm{m}$ and $75 \mu \mathrm{m}$ respectively. Both the DFB and the EAM have $50 \Omega$ resistors for impedance matching. The DEML chip was fixed on a ceramic sub-mount (size $2 \mathrm{~mm} \times 6 \mathrm{~mm} \times 0.5 \mathrm{~mm}$ ), with a temperature sensor $\left(\mathrm{R}_{\mathrm{NTC}}\right)$ placed beside the chip, and a lensed fiber faced with the EAM output. A circuit board was assembled to provide independent radio frequency (RF) inputs to the DFB and the EAM. A side scheme of the DEML, and a picture of the assembly are presented in Fig. 2(a) and Fig. 2(b) respectively. 


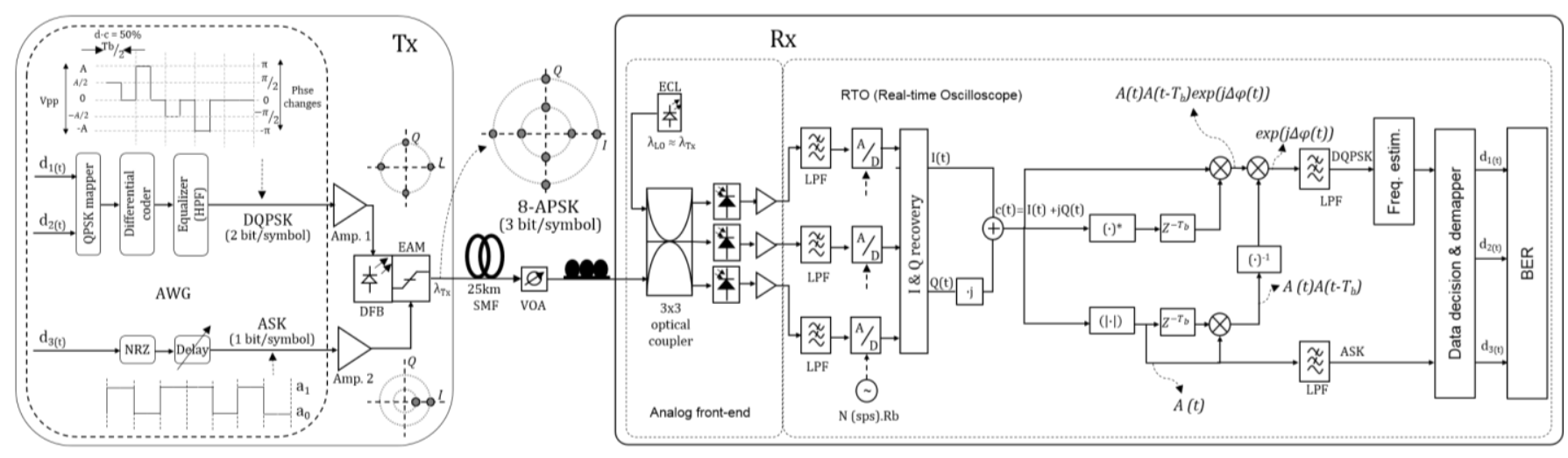

Fig. 1. Experimental setup. The insets show the modulating waveforms for the DFB and EAM sections, as well as the I-Q diagram for the DQPSK, ASK and 8-APSK transmitted optical signal.

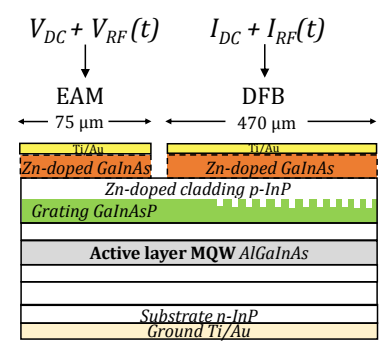

(a)

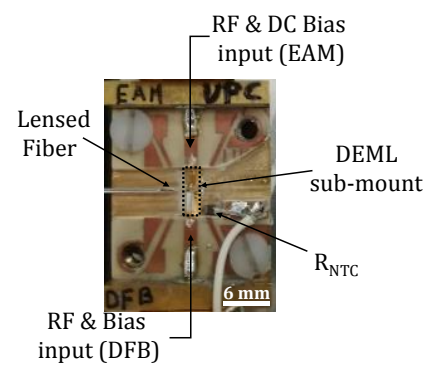

(b)
Fig. 2. (a) Side-scheme of DEML; (b) Assembled sub-mount and DEML.

An external temperature control was used to keep an operative condition in the chip of $26^{\circ} \mathrm{C}$. The DFB wavelength was $\lambda_{T X}=$ $1556.16 \mathrm{~nm}$. We measured the static characteristics of the DFB and the EAM. The results are plotted in Fig. 3. The DFB threshold current is as low as $7 \mathrm{~mA}$. The DFB was operated at bias current of $60 \mathrm{~mA}$ (a balance between wavelength stability, bandwidth (BW) and output power). The coupled optical power is limited by the modulator effective insertion loss, which results from the choice of the EAM bias point. When a static bias of $-2 \mathrm{~V}$ was applied to the EAM section, the output power of the DEML was $0 \mathrm{dBm}$. The modulation BW of the DFB is expected to be about $10 \mathrm{GHz}$, and even larger for the EAM [8]. However, in our setup and due to constraints of the RF circuitry (i.e. cable lengths, bondings, and parasitic packaging effects) the effective modulation BW was limited to $2 \mathrm{GHz}$ and $4 \mathrm{GHz}$ for the DFB and the EAM respectively. In addition, the measured DFB linewidth was $300 \mathrm{KHz}$.

A lensed fiber was faced and properly aligned with the EAM output of the DEML. An optical isolator was fused to the lensed fiber for minimizing optical reflections. The optical output signal was sent through a $25 \mathrm{~km}$ single mode fiber (SMF) link. Afterwards, a variable optical attenuator (VOA) emulated the splitting losses of the passive optical network (PON), and limited the power into the Rx. A polarization controller compensated the fluctuations in the state of polarization caused by the fiber.

The $\mathrm{Rx}$ was based on a coherent intradyne scheme. A 3x3 optical coupler mixed the incoming signal with the local oscillator (LO), that was a $100 \mathrm{kHz}$ linewidth external cavity laser (ECL) emitting at $3 \mathrm{dBm}$ and tuned to similar Tx wavelength $\lambda_{T X} \approx \lambda_{L O}$.

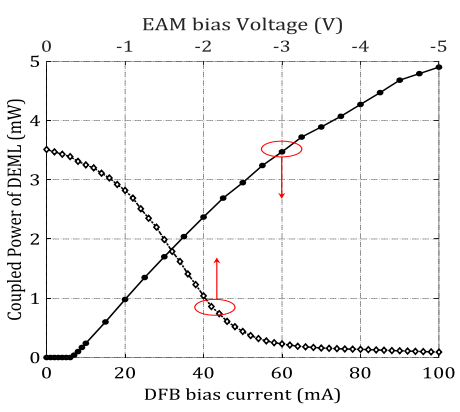

Fig. 3. Static characteristics: $\mathrm{DFB}\left(\mathrm{V}_{\mathrm{EAM}}=0 \mathrm{~V}\right)$, EAM $\left(\mathrm{I}_{\text {bias } \mathrm{DFB}}=60 \mathrm{~mA}\right)$.

The optical signals at the coupler outputs were detected with three $10 \mathrm{GHz}$ p-i-n photodiodes (PDs) followed by low-noise transimpedance amplifiers and then low-pass filtered. The three photodetected currents were sampled and processed by a real-time oscilloscope. Next, in-phase $(I)$ and quadrature $(Q)$ components of the complex signal $(c(t))$ were obtained as described in [9]. The DQPSK $(\exp (j \Delta \varphi(t)))$ and ASK data $(A(t))$ were simultaneously demodulated. ASK demodulation was done with an envelope detector. The DQPSK data were differentially demodulated by multiplying with the complex conjugate of a symbol period (Tb) delayed signal. In order to cancel the amplitude component from the DQPSK signal, the recovered ASK data were multiplied by a symbol-period delayed version of itself $\left(A\left(t-T_{b}\right)\right)$, then inverted, and multiplied by the recovered DQPSK data. Finally the DQPSK and the ASK data were low-pass filtered before data decision. The symbols were demapped and the bit error ratio (BER) computed over the three bit streams.

We first tested the direct DQPSK modulation at 1.25 Gbaud and 2.5 Gbaud. The EAM was unbiased and the DFB was phase modulated with a proper digital signal of duty-cycle $=50 \%$ and peak to peak voltages of $V p p=0.64 \mathrm{~V}$ and $V p p=1.48 \mathrm{~V}$ respectively. In optical back to back (btb), we achieved Rx sensitivities of $-49 \mathrm{dBm}$ and $-46.5 \mathrm{dBm}$ at second generation FEC limit of BER $=4 \times 10^{-3}[10]$. The results are plotted in Fig 4 .

Afterwards, we tested the ASK modulation at the same baud rates. We biased the EAM at $-2.2 \mathrm{~V}$ and we tested the performance for three different extinction ratio (ER) values of 2, 3.8 and $5.1 \mathrm{~dB}$. 


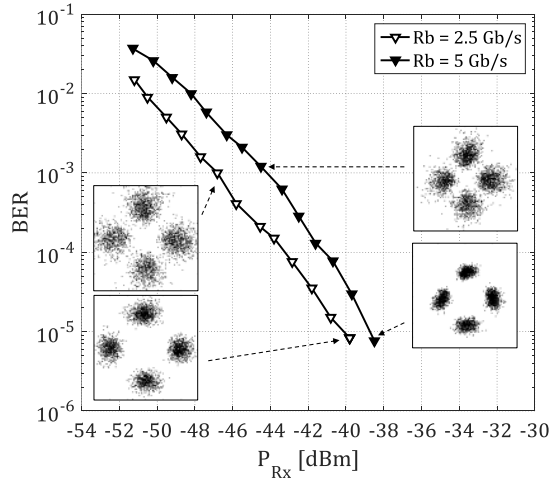

Fig. 4. BER against Rx power for DQPSK modulation at 1.25 Gbaud ( $R b$ $=2.5 \mathrm{~Gb} / \mathrm{s})$ and $2.5 \mathrm{Gbaud}(R b=5 \mathrm{~Gb} / \mathrm{s})$. The insets show the I-Q diagrams of the recovered symbols at $\mathrm{BER}=10^{-5}$ and $\mathrm{BER}=10^{-3}$.

The measured BER against the Rx optical power at 1.25 Gbaud and 2.5 Gbaud are represented by the curves of Fig. 5(a) and Fig. 5(b) respectively. The achieved Rx sensitivities at the same FEC limit of $\mathrm{BER}=4 \times 10^{-3}$ are presented in table 1 .

Table 1. Rx sensitivity for ASK modulation at BER $=4 \times 10^{-3}$

\begin{tabular}{cccc}
\hline & \multicolumn{3}{c}{ ER $(\mathrm{dB})$} \\
\cline { 2 - 4 } $\mathrm{Rb}(\mathrm{Gb} / \mathrm{s})$ & 2 & 3.8 & 5.1 \\
\hline 1.25 & $-46.5 \mathrm{dBm}$ & $-49 \mathrm{dBm}$ & $-51 \mathrm{dBm}$ \\
2.5 & $-43 \mathrm{dBm}$ & $-46.5 \mathrm{dBm}$ & $-48.5 \mathrm{dBm}$ \\
\hline
\end{tabular}

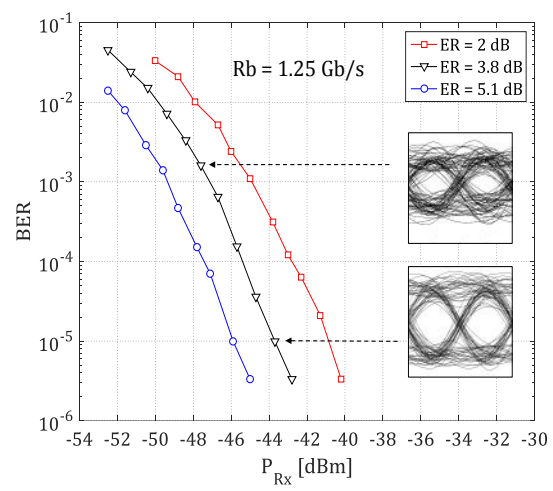

(a)

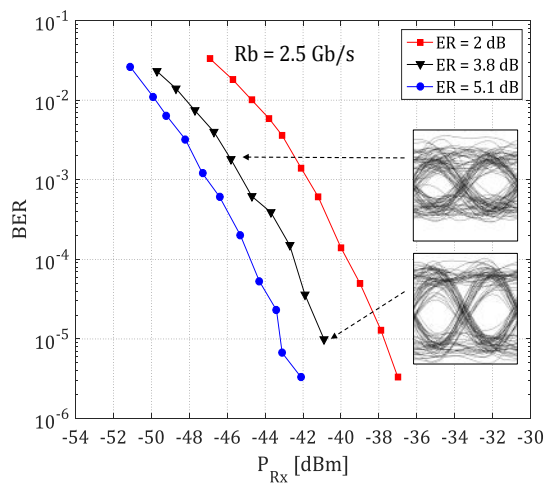

(b)

Fig. 5. BER against Rx power for ASK modulation at: (a) 1.25 Gbaud $(R b=1.25 \mathrm{~Gb} / \mathrm{s})$ and (b) $2.5 \mathrm{Gbaud}(R b=2.5 \mathrm{~Gb} / \mathrm{s})$. The insets show the eye diagrams at $\mathrm{BER}=10^{-5}$ and $\mathrm{BER}=10^{-3}$ for $\mathrm{ER}=3.8 \mathrm{~dB}$.
Finally, we combined DQPSK and ASK formats and we tested the proposed differential 8-APSK modulation. Ideally, for the DEML, we would like to consider the laser as a pure frequency modulator and the EAM as an amplitude modulator. However, this is not the case, and a strict evaluation of the device chirp is required [8]. Therefore, we performed both small and large signal evaluation of the DFB and EAM chirp characteristics, using an optical filter as frequency discriminator [11].

For the DFB laser the optical phase variation is related with the frequency chirp by [12]:

$$
2 \pi \Delta v(t)=\frac{d \phi(t)}{d t}=\frac{\alpha_{H}}{2}\left(\frac{1}{P(t)} \frac{d P(t)}{d t}+\kappa P(t)\right)
$$

where $P(t)$ is the signal power, $\alpha_{H}$ is the linewidth enhancement factor or Henry coefficient, and $\kappa$ is the adiabatic chirp coefficient. From Eq. (1) we observe that the phase variations depend on both the power and the duty-cycle of the signal, which can be digitally adjusted [7]. At mid frequencies $(10 \mathrm{MHz}<f<5 \mathrm{GHz})$ the second term of Eq. (1), i.e. the adiabatic chirp, prevails. For larger frequencies $(f>5 \mathrm{GHz})$ the first term of Eq. (1) i.e. the transient chirp, is dominant and the frequency chirp gets proportional to the modulation frequency. The thermal effects are not considered in Eq. (1) since there are only significant at lower frequencies $(f<10$ $\mathrm{MHz}$ ) [13]. The $\alpha_{H}$ and $\kappa$ parameters can be found from the relation between the amplitude of the intensity modulation (IM) and frequency modulation (FM) responses, both measured using a vectorial network analyzer, and considering that [14]:

$$
\lim _{f m \rightarrow \infty} \frac{2 \beta}{m}=\left|\alpha_{H}\right|
$$

where $\beta$ and $m$ are the frequency and intensity modulation indexes respectively. We measured $\alpha_{H}$ for different DFB bias currents and when the EAM was unbiased. The sign of $\alpha_{H}$ is given by the phase of the IM and FM responses ratio [14]. The results plotted in Fig. 6, show that for the DFB, $\alpha_{H}$ is approximate equal to 2 , a value that is not strongly dependent on the laser bias current. Considering the adiabatic chirp regime, we obtained $\kappa=1.08 \times 10^{13} \mathrm{~Hz} / \mathrm{W}$, for a DFB bias current of $60 \mathrm{~mA}$. This value is proportional to the photon density and increases with the bias current [11].

For the EAM, the optical phase variation is exclusively dependent on the transient chirp, then we considered only the first term of Eq. (1). Conversely to the DFB case, the value of $\alpha_{H}$ parameter for the EAM is strongly dependent on the applied bias voltage, ranging approximately from -1.2 to 2 . This behavior is shown in Fig. 6 for a fixed DFB bias current of $60 \mathrm{~mA}$. As we desired to perform the phase modulation with the DFB, we needed to reduce the chirp inherent to the EAM. Hence, we properly biased the EAM around the zero-chirp point observed in Fig.6 $\left(V_{\text {EAM }} \approx-2.2 \mathrm{~V}\right)$. In addition, we set the ASK modulation to have an $\mathrm{ER}=3.8 \mathrm{~dB}$, because it represented the symmetric Euclidean distance among the constellations points of the 8-APSK signal.

Next, the DFB was directly modulated in phase, and synchronously the amplitude of the optical signal was modulated with the EAM. Fig. 7 shows the BER against the Rx optical power. The sensitivities for BER $=4 \times 10^{-3}$ were $-45.5 \mathrm{dBm}$ and $-43.5 \mathrm{dBm}$ at $3.75 \mathrm{~Gb} / \mathrm{s}$ and $7.5 \mathrm{~Gb} / \mathrm{s}$ respectively. Notably, when transmitting through $25 \mathrm{~km}$ of SMF, the measured penalty was only $1 \mathrm{~dB}$, and we obtained an Rx sensitivity of $-42.5 \mathrm{dBm}$ at $7.5 \mathrm{~Gb} / \mathrm{s}$. Under laser direct phase modulation, the achieved results improve in 
approximately $4 \mathrm{~dB}$ the performance of similar experimental test with 8-ary modulation formats like 8-DPSK [15]. This is because the Euclidean distance between the constellations points of the 8APSK Tx is larger than in 8-DPSK for the same mean power. Additionally, we used as LO an ECL with narrower linewidth than the DFB laser used in [15].

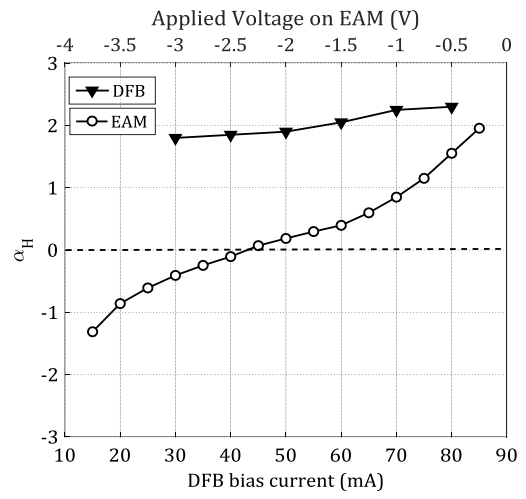

Fig. 6. Measurements of $\alpha_{H}$ parameter for the DFB $\left(\mathrm{V}_{\text {EAM }}=0 \mathrm{~V}\right)$ and the EAM (l bias DFB $=60 \mathrm{~mA})$

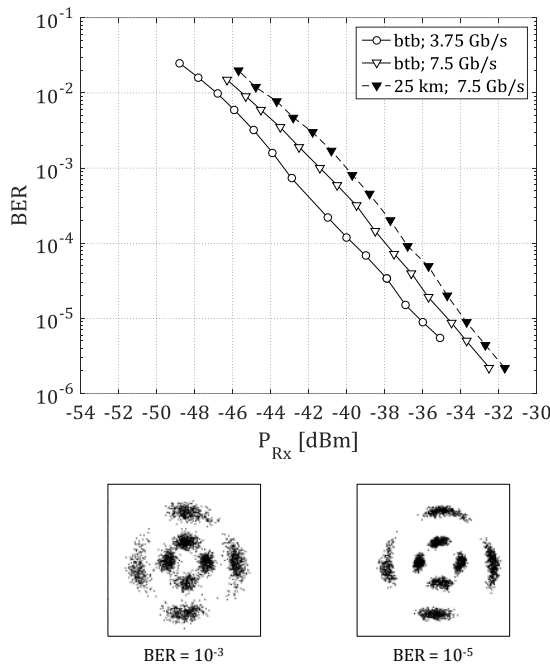

Fig. 7. BER against Rx power for differential 8-APSK at $1.25 \mathrm{Gbaud} / \mathrm{s}$ $(R b=3.75 \mathrm{~Gb} / \mathrm{s})$ and $2.5 \mathrm{Gbaud} / \mathrm{s}(R b=7.5 \mathrm{~Gb} / \mathrm{s})$. The I-Q diagrams show the recovered symbols at $\mathrm{BER}=10^{-3}$ and $\mathrm{BER}=10^{-5}$.

Despite the improved performance, there are two limitations that are worth mentioning. First, although we would like to consider both devices independently, they appear not to be totally uncorrelated [8]. When the EAM is modulated, the wavelength slightly fluctuates and shows drifts mainly due to temperature variations in the chip. This is because the fast amplitude transitions in the EAM affect the temperature due to the thermal heating drift in the resistor matching the EAM to $50 \Omega$. To overcome this problem we applied a differential $\mathrm{m}^{\text {th }}$ power algorithm at the $\mathrm{Rx}$ DSP for frequency estimation [16], to compensate the frequency drifts caused by the temperature instabilities in the chip. Second, an additional frequency chirp is caused by the optical feedback from the front faced (modulator site) and is dependent on the modulator bias $[8,14]$. This contribution can be noted in the slight widening of the recovered symbols as shown in the I-Q diagram of Fig (7). To minimize the penalty, especially at lower signal-to-noise ratios (SNR), additional optical isolation is needed [17], as well as some degree of equalization at the Rx should be investigated.

In conclusion, we propose and experimentally demonstrate, for the first time to the best of our knowledge, a simple externalmodulator-free differential 8-APSK $\mathrm{Tx}$ by a monolithically integrated DEML. The transmitted signal was detected with an intradyne coherent $\mathrm{Rx}$, using both, the amplitude and phase of the optical signal. Compared with the conventional ASK or DQPSK modulation, the spectral efficiency increases by 3-times and 1.5times respectively, achieving a Rx sensitivity of $-42.5 \mathrm{dBm}$ at 7.5 $\mathrm{Gb} / \mathrm{s}$. These results show the feasibility for a simple, small footprint and low cost external-modulator-free Tx, able to support flexible transmission capacity, which are important requirements in upcoming $5 \mathrm{G}$ era.

Funding. Spanish Ministry of Economy and Competitiveness (MINECO) FLIPER (TEC2015-70835); Agency for Management of University and Research Grants (AGAUR) (2016FI_B 00758).

Acknowledgment. We thank III-V lab, for supplying the DEML.

\section{References}

1. M. Nekovee, Y. Wang, M. Tesanovic, S. Wu, and M. Al-Imari, J. Commun. Inform. Netw. 1, 44 (2016).

2. R. M. Ferreira, J. D. Reis, S. M. Rossi, S. B. Amado, F. P. Guiomar, A. Shahpari, J. R. F. Oliveira, A. N. Pinto, A. L. Teixeira, J. Lightwave Technol. 34, 826 (2016).

3. M. Presi, M. Artiglia, F. Bottoni, M. Ranello, I. N. Cano, J. Tabares, J. C. Velásquez, S. Ghasemi, V. Polo, G. Y. Chu, J. Prat, G. Azcarate, R. Pous, C. Vilà, H. Dabregeas, G. Vall-llosera, A. Rafel, and E, Ciaramella, J. Lightwave Technol. 35, 5220 (2017).

4. C. Kazmierski, J. Opt. Commun. Netw. 8, A8 (2012).

5. J. C. Velásquez, M. Domingo, V. Polo and J. Prat, in Optical Fiber Communication Conference 2018 (Optical Society of America, 2018), paper M3B.4.

6. I. N. Cano, A. Lerín, and J. Prat, IEEE Photon. Technol. Lett. 26, 35 (2016).

7. J. C. Velásquez, I. N. Cano, V. Polo, M. Domingo, and J. Prat, IEEE Photon. Technol. Lett. 30, 137 (2018).

8. D. Erasme, T. Anfray, M. E. Chaibi, K. Kechaou, J. Petit, G. Aubin, K. Merghem, C. Kazmierski, J-G Provost, P. Chanclou, and C. AupetitBerthelemot, J. of Lightwave. Technol. 32, 4068 (2014).

9. C. Xie, P. J. Winzer, G. Raybon, A. H. Gnauck, B. Zhu, T. Geisler, and B. Edvold, Opt. Express 20, 1164 (2012).

10. T. Mizuochi, in Optical Fiber Communication Conference 2008 (Optical Society of America, 2008), paper OTuE5.

11. K. Sato, S. Kuwahara, and Y. Miyamoto, J. Lightwave Technol. 23, 3790 (2005).

12. D. Che, Q. Hu, F. Yuan, Q Yang, and W. Shieh, IEEE Photon. Technol. Lett. 27, 2407 (2015).

13. J.-G. Provost and F. Grillot, IEEE Photon. J. 3, 476 (2011).

14. P. Brosson and H. Bissessur, IEEE J. Sel. Top. Quantum Electron. 2, 336 (1996)

15. I. N. Cano, J. C. Velásquez, and J. Prat, in Optical Fiber Communication Conference 2016 (Optical Society of America, 2016), paper M3C.4.

16. J. Tabares, S. Ghasemi, V. Polo, and J. Prat, J. Lightwave Technol. 36, 2941 (2018).

17. N. H. Zhu, G. H. Hou, H. P. Huang, G. Z. Xu, T. Zhang, Y. Liu, H. L. Zhu, L. J. Zhao, and W. Wang, J. Quam. Electron. 43, 535 (2007). 